\title{
DRAG OF MICRO-PARTICLES BY AN EXTENDED NEMATIC-ISOTROPIC INTERFACE
}

John L.West, ${ }^{*}$ Ke Zhang, and Anatoliy Glushchenko

Liquid Crystal Institute, Kent State University, Kent,

Ohio 44242, USA

Yuri Reznikov

Institute of Physics, Prosect Nauky 46, Kyiv 03039, Ukraine

\author{
Denis Andrienko \\ Max-Plank Institute for Polymer Research, Ackermannweg 10, \\ Mainz 55128, Germany
}

\begin{abstract}
We studied the behaviour of polymer particles in a moving interface between the nematic (N) and isotropic (I) phases of a nematic liquid crystal (LC). We showed that the NI-interface is extended (E) and has a layered N-I-N structure in the vertical cross-section of the sample; the wedge of the isotropic phase is bounded by the nematic phase, which is limited by the cell substrates. The minimum of the cell free energy defines the position of particles in the interface region. We find that the preferable position of the particle is at the vertex of the wedge formed by the isotropic phase. The particles are captured by the vertex line and follow the interface when it moves.
\end{abstract}

Keywords: drag effect; nematic-isotropic liquid interface; nematic liquid crystal; polymer particles

\section{INTRODUCTION}

Precise methods of manipulation and separation of tiny particles in liquids are one of fascinating achievements of modern hi-tech. Similar bacterial

The authors wish to acknowledge Victor Reshetnyak and Andrey Iljin for stimulating discussions and valuable suggestions. The work was partially supported by DARPA grant \#444226 and by the grant "Composite liquid crystal and polymer materials for information technologies" of National Academy of Science of Ukraine.

*Corresponding author. Tel.: +1 330672 2581, Fax: +1 330672 2796, E-mail: johnwest@ lci.kent.edu 
species and alive cells can be separated by using dielectrophoretic effects [1-3]. Combination of electrophoretic and dielectrophoretic forces allows trapping and delicate manipulating DNA and polymer particles on planar microelectrodes [4,5]. Effects of dielectric particles manipulation by an electric field lie in a base of operation of $e$-papers and electrophoretic displays $[6,7]$.

Recently it was found that not only external electric forces but also a boundary between two thermodynamically stable phases can effectively organize and separate tiny particles. Slow evaporation of dispersion solvent leads to perfectly organized colloid and photonic crystals $[8,9]$. Micro-particles can be effectively positioned and dragged by the boundary between the liquid phases of different symmetry. We found drag effect on colloidal particles by a moving interface between isotropic and nematic phase of liquid crystal (LC) [10]. This effect is mainly due to the surface tension effects and long-range distortions of the director around the colloidal particle when it is in the nematic phase. To minimize the director distortions, the nematic phase expels particles into the isotropic phase and the moving interface accumulates particles. We demonstrated that, depending on the speed of the interface movement, particle size, and elastic properties of LC, one can move particles of specified radius and control the spatial distribution of these particles in the cell.

In the Reference [10] it was suggested that particles are dragged by a flat interface between the nematic and isotropic phases. However, as was shown by Oswald et al. [11], the moving interface has rather complicated, extended, shape of a meniscus of nematic phase extended into isotropic phase. Therefore, to study the drag on a particle, we shall consider interaction of the particle not just with the flat $N I$-interface but rather with a complex two-phase region - extended interface.

Here we report a first investigation of behaviour of small polymer particles in a moving extended NI-interface. We show that under typical experimental conditions the extended NI-interface has a wedge-sandwich structure, parameters of which control the drag of the particles.

\section{EXPERIMENTS}

In our experiments we mostly used the parallel cells with planarly or homeotropicaly oriented LC 5CB. The planar alignment was provided by a rubbed layer of polyimide PI2555 coating and the homeotropic alignment was achieved by coating with polyimide Nissan 7511L.

Thickness of the cells, $L$, was $40 \mu \mathrm{m}$. In some experiments we also used the wedged cells with the wedge angle $\sim 0.3^{\circ}$ and the thickness in the 
range $0 \sim 100 \mu \mathrm{m}$. Spherical polystyrene particles were dispersed in the LC in the isotropic phase in ultrasonic shaker. The diameter of the particles was $d=16 \mu \mathrm{m}$; their density, $\rho=1.04 \mathrm{~g} / \mathrm{cm}^{3}$, was slightly more than the density of $5 \mathrm{CB}\left(\rho=1.007 \mathrm{~g} / \mathrm{cm}^{3}[12]\right)$. The surface of the particles provided planar alignment of $5 \mathrm{CB}$.

Sketch of the experimental set-up is shown in Figure 1. One end of the cell was placed on a hot-stage which temperature was kept with the accuracy $0.05^{\circ} \mathrm{C}$ and could be changed with the step of $0.1^{\circ} \mathrm{C} / \mathrm{min}$. The other end was put on a metal plate, kept at room temperature. The edges were bonded to the hot-stage and metal plate by a thermo-conductive paste to provide a homogenous thermal contact. By changing the temperature of the hot-stage we were able to control the temperature gradient and change the temperature along the long side of the cell. The characteristic temperature gradients over the cell were $20 \sim 30^{\circ} \mathrm{C} \mathrm{cm}^{-1}$. The speed of the temperature changes at the hot stage was in the range $0 \sim 5^{\circ} \mathrm{Cmin}^{-1}$. Changing the temperature of the hot stage resulted in moving the interface between the isotropic and nematic phases with the speed, $v=$ $0 \sim 25 \mu \mathrm{m} \mathrm{s}^{-1}$. We observed behaviour of the particles at the moving interface through the polarizing microscope.

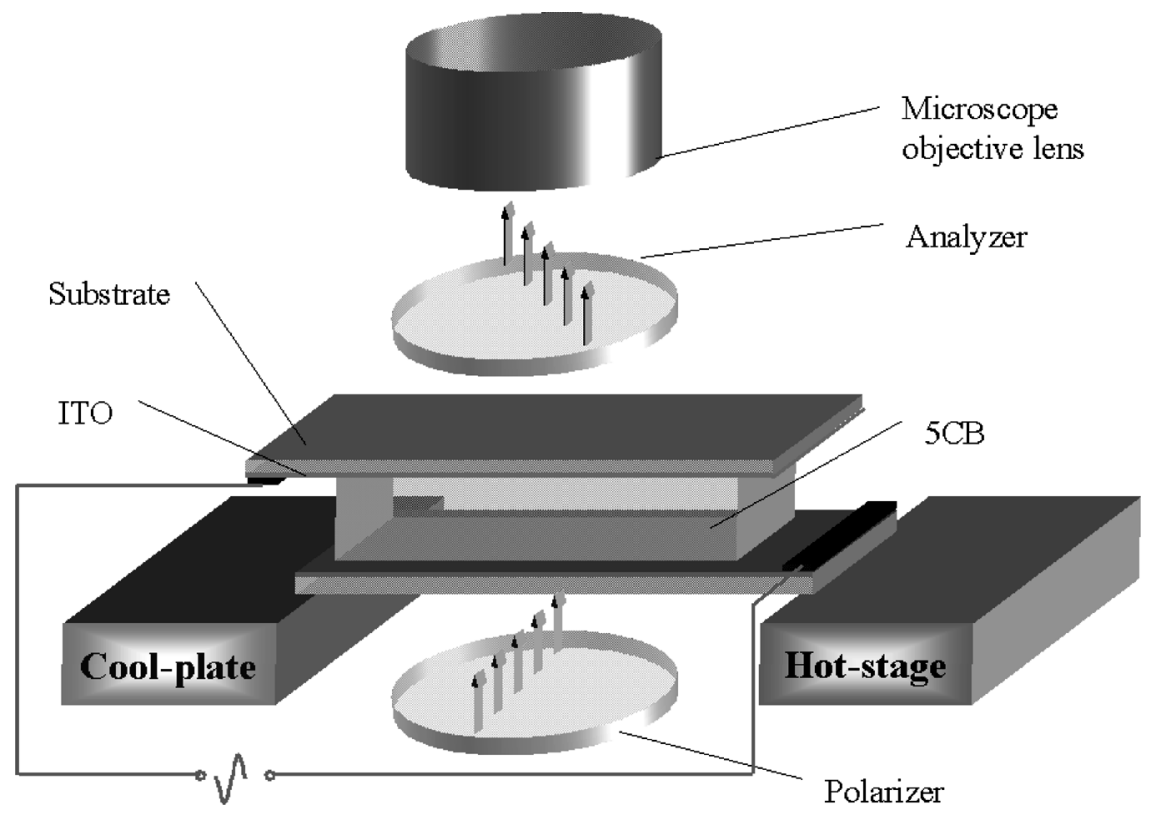

FIGURE 1 Experiment set up. 


\section{RESULTS AND DISCUSSION}

Typical optical textures of the LC cell in the region of the transition between the nematic and isotropic phases are presented in Figure 2a-c. One can see that the areas of homogenous nematic and isotropic phases are separated by a rather wide birefringent region - extended $N I$-interface. The temperature and width of the extended interface depended on the
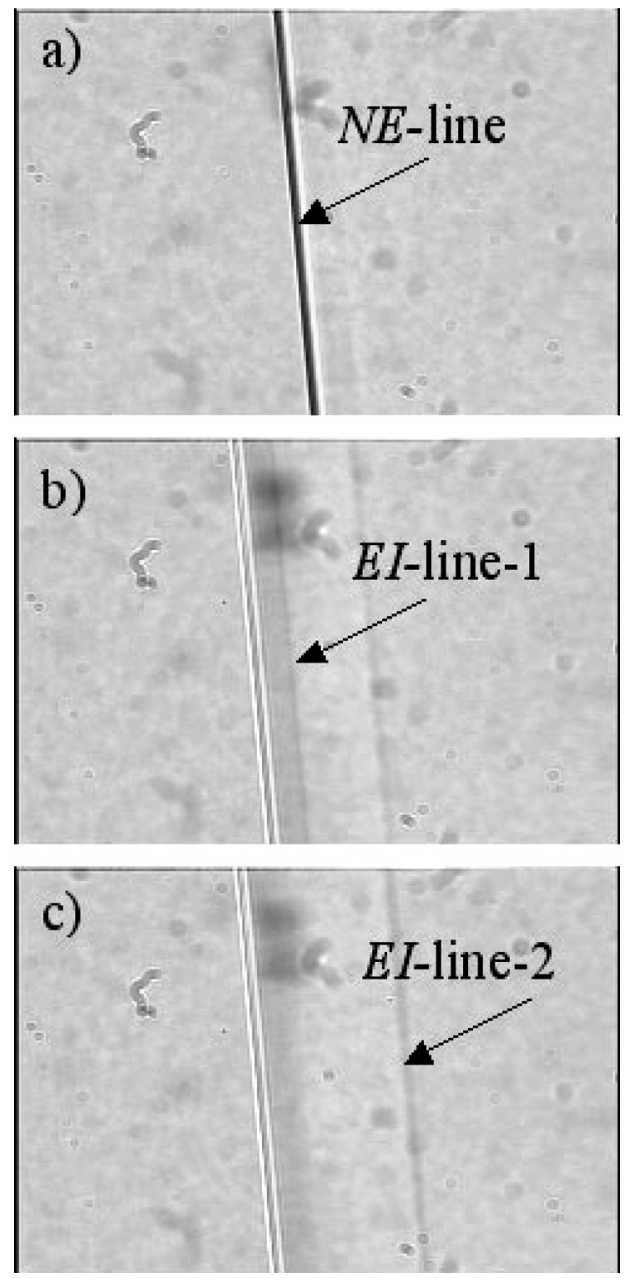

FIGURE 2 The textures of the extended interface. a) the $N I$-line is in focus at the bulk of the LC; b) the first EI-line is in focus at the bottom substrate; c) the second $E I$-line is in focus at the top substrate. 
speed of the interface movement. The width of the extended interface, $\Delta T_{n-i}$, in the stationary state $(v=0)$ was about $0.025^{\circ} \mathrm{C}$ which corresponds to $\sim 9 \mu \mathrm{m}$ in spatial units. The moving interface was much wider and its width was increasing with the speed; at $v=25 \mu \mathrm{m} \mathrm{s}^{-1}$ it was $\Delta T_{n-i} \sim 0.1^{\circ} \mathrm{C}(30 \mu \mathrm{m})$. From the side of the nematic phase, the extended interface region was bound by a director distortion line ( $N E$-line); the boundary with the isotropic phase (EI-line) was not so distinguished. To scan the structure of the extended interface, we moved the lens of the microscope with the small sharpness deepness $(\sim 2 \mu \mathrm{m})$ perpendicular to the cell plane and observed the textures through the cell thickness $(\sim 40 \mu \mathrm{m})$. By this mean we found that the $N E$-line (Fig. 2a) was located in the bulk of the LC cell and shifted from the middle line toward the bottom substrate on several microns. We also found two EI-lines; one EI-line was located at the bottom substrate (Fig. 2b), and the other one - on the top substrate (Fig. 2c). These lines were located at different distance from the $N E$-line.

In most part of the interface a homogeneous structure with the optical axis in a plane parallel to the rubbing direction was observed (Fig. 3a). The birefringence of this region decreases towards the boundary with isotropic phase. Next to the $N E$-line we found a region that looked bright between crossed polarizers on the background of the remaining, dark interface (Fig. 3b). We concluded that, in this region, the optical axis twists, escaping the plane parallel to the rubbing direction. The value of twist angle was not well reproduced and lay between $45-90^{\circ}$.
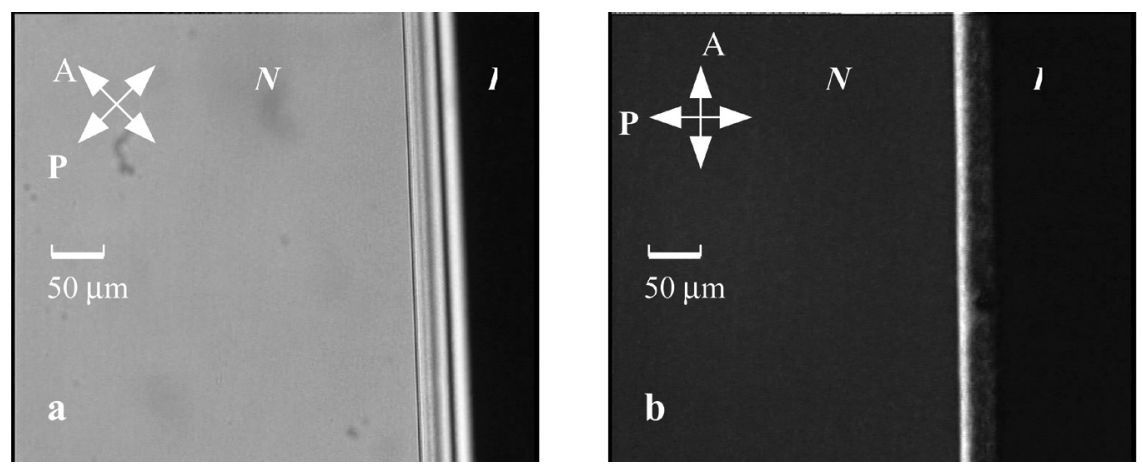

FIGURE 3 Snap shot of N/I interface moving across in a planar cell when cooling speed of hotstage is $5^{\circ} / \mathrm{min}$. The orientations of the polarizer $(\mathbf{P})$ and the analyzer (A) are marked by the white arrows. The direction of the rubbing of the cell substrates is parallel to the horizontal side of the pictures. 
The observations above allowed us to propose a model of the extended interface and reconstruct its structure. A nematic-isotropic transition is a weak 1st-order transition, and the presence of impurities results in temperature region, $\Delta T_{n-i}$, of coexisting of nematic and isotropic phases [13-15]. Value of $\Delta T_{n-i}$ is proportional to the density of impurities in $L C$ and can be rather wide (0.1-1deg) (Fig. 4). A change of the temperature in the two-phase region results in redistribution of volumes $V_{\text {nem }}, V_{i s o}$, of nematic $(N)$ and isotropic (I) phases due to redistribution of impurity concentrations between the phases. The volume of the isotropic phase grows with the temperature at the expense of nematic phase. The ratio $V_{n e m} / V_{i s o}$ changes with temperature according to the level rule:

$$
\frac{V_{\text {nem }}}{V_{\text {iso }}}=\left|\frac{c_{\text {iso }}-c_{\text {total }}}{c_{\text {total }}-c_{\text {nem }}}\right|
$$

where, $c_{i s o}, c_{\text {nem }}$, are the equilibrium impurity densities of isotropic and nematic phase, and $c_{\text {total }}=c_{i s o}+c_{\text {nem }}$. Because the nematic phase wets the polyimide surface better than the isotropic one, a slow entrance to

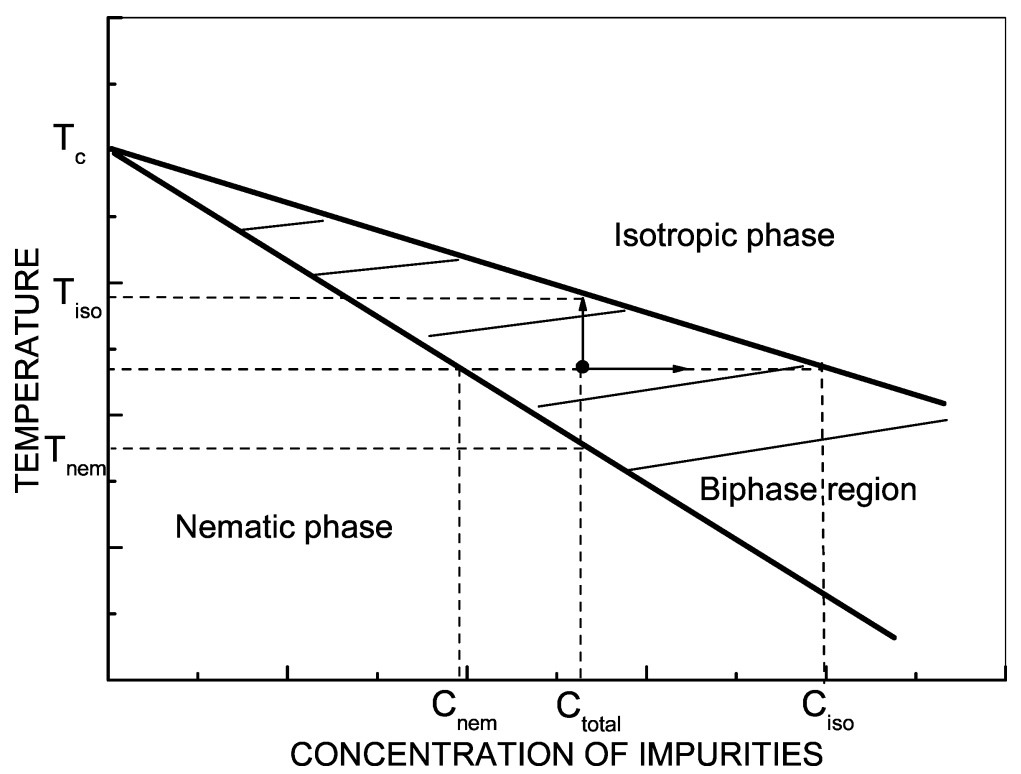

FIGURE 4 Phase diagram of impurity nematic LC. The values $\Delta T$ and $\Delta C$ determine the shift of the operation point $\left(T_{0}, c_{\text {total }}\right)$ at heating and changes of impurity concentration, respectively. 
the region of the coexistence of nematic and isotropic phases resulted in a sandwich $N-I-N$ structure $[15,16]$. According to the level rule, the thickness of the isotropic layer increases with temperature. Therefore, in zero approximation the extended interface should have a wedge shape in temperature gradient; the wedge of the isotropic phase is bounded by the nematic phase, limited by the cell substrates (insertion at Fig. 5). While the interface moves, the steady distribution of the concentration of impurities between nematic and isotropic phases is disturbed resulting in broadening of the extended interface.

The described simple picture does not take into account the elasticity of LC. The shape of the interface is defined by the minimum of the interface energy, which depends on the director alignment at the interface. As was shown by Faetti [17], a degenerated conic alignment of 5CB with the tilt angle $\theta=26.5^{\circ}$ and rather strong anchoring energy $\left(\mathrm{W} \sim 10^{-3} \mathrm{erg} \mathrm{cm}^{-2}\right.$ ), is realised at the $N I$-boundary. It causes deformations of the director in the extended interface which is most strong at the vertex of the isotropic wedge. It is reasonable to suggest that in order to minimize these deformations, the interface bends nearby of the vertex, adapting to the planar

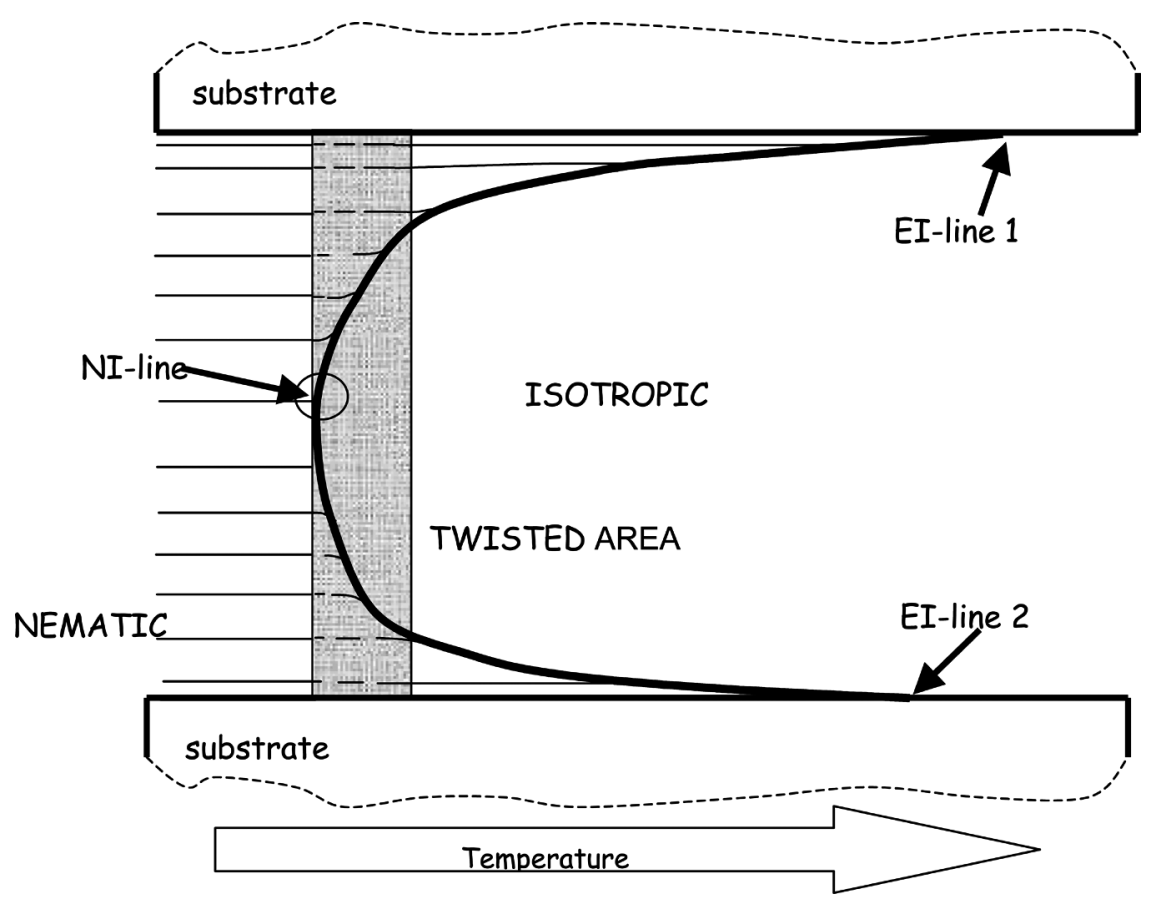

FIGURE 5 The Structure of the extended interface. 
boundary conditions at the cell boundaries and at the interface itself, minimizing the director deformations. Therefore, the structure of extended interface has a meniscus shape (Fig. 5), similar to reported by Oswald [11]. The vertex of the meniscus forms the sharp $N E$-line at the middle of the cell. The deformation of the director and optical retardation in the vicinity of the EI-lines are much less that result in their indistinctive look. A slight asymmetry of the shape of the extended interface is a consequence of the weak temperature gradient in the plane perpendicular to the cell substrates.

The orientation of the director in the most part of the extended interface is determined by the direction of rubbing, $\vec{e}_{\text {rub }}$. The azimuthal turn of the director from $\vec{e}_{r u b}$ nearby of the $N E$-line (the azimuthally oriented area) can be explained by the effect of the geometrical anchoring. As was shown by Lavrentovich [18], in the general case of nonparallel bounding, a particular value of the azimuthal orientation of LC may be energetically preferable, that is our case. Indeed, nearby the $N E$-line the LC is placed between two highly tilted aligning surfaces - the rigid substrate surface and the liquid $N I$-surface. As was said before, the rigid substrate imposes a planar alignment to LC and the $N I$-surface provides a degenerated conic alignment of LC with $\theta=26.5^{\circ}$. According to [18], in this geometry, at zero anchoring and at the angle between bonded surfaces, $\gamma>\theta$, the surfaces tilt leads to the director turn out from $\vec{e}_{\text {rub }}$ in azimuthal direction. Since the order parameter of nematic phase in the extended interface is minimal, it is reasonable to suggest that the anchoring is very weak in our case. Therefore, the tilt between NI-surface and cell substrates is more than $26.5^{\circ}$ in the azimuthally oriented area and $\gamma \approx 26.5^{\circ}$ at the border of this area.

The moving interface dragged the particles dispersed in the LC. Behaviour of the particles inside of the interface was rather complicated; it strongly depended on the speed of the interface and on the sign of the temperature change (cooling or heating).

In the case of the hot-stage cooling, the interface passed toward the hot-stage and met the particles from the side of the $I E$-lines. At the high speed of the interface movement $\left(v_{\text {int }} \geq 18 \mu \mathrm{m} / \mathrm{s}\right.$ ) the interface did not affect the particles position that is the drag effect was not observed. At $v_{\text {int }} \leq 18 \mu \mathrm{m} / \mathrm{s}$ the particles did not move till they are at the definite distance, $d_{c r}$ (around half of the interface extent) from the $N E$-line. By achieving this distance, the particles were attracted by the $N E$-line, e.g., the particles moved to the $N E$-line much faster than the interface passes by in opposite direction. Finally, the particles were captured by $N E$-line and moved together with this line toward the hot-stage. At a very low speed $\left(v_{\text {int }} \leq 3 \mu \mathrm{m} / \mathrm{s}\right)$ the particles were attracted by the $N E$-line but they are not captured by the line; after touching the line, the particles 
bounced back to the interface and attracted to the $N E$-line again. This process repeated again and again; it looked like the $N E$-line dribbles the particles.

At the heating of the hot-stage, the interface moved away from the hotstage and the particles met it from the side of the $N E$-lines. In this case the particles were attracted by the $N E$-line being at the distance comparable with their diameter. At $v_{\text {int }} \leq 4 \mu \mathrm{m} / \mathrm{s}$ the particles were captured by the $N E$-line and moved together. At $v_{\text {int }} \geq 4 \mu \mathrm{m} / \mathrm{s}$ the $N E$-line was passing by the particles.

Reach dynamics of particles close to the moving interface is partially connected to the complex interaction of a single particle with the interface. To calculate the trajectory of a moving particle in a nematic with a non-uniform director field is a challenging task, even if we omit the hydrodynamic effects. When we have two different phases and a complex $N-I-N-$ structure the problem is much more difficult to solve, even numerically. Here we present only some qualitative explanations of the experimental results.

First, we consider the situation when the particle is in the isotropic phase and the nematic phase moves towards it (hot-stage cooling). Since $\rho_{\text {part }}>\rho_{L C}$, the particle is usually located close to the bottom substrate. Therefore, it first meets the interface somewhere close to the EI-line. There the interface captures the particle, because the free energy of the system has a minimum when the particle is at the interface [to be published]. In addition, elastic forces expel the particle from the nematic phase, towards the isotropic phase. These forces are directed normal to the interface, and, since the interface is tilted with respect to the substrates, there is a component of the force pushing the particle to the edge of the isotropic meniscus ( $N E$-line). Moreover, next to the $N E$-line we have the strongest deformations of the director, even with possible topological defects at that point [11]. Since the particle tries to occupy the space with the maximal distortions of the director, in order to minimize the elastic energy of the director deformations [19], there is an additional contribution to the force pushing the particle towards the $N E$-line. Finally, the particle settles at the $N E$-line and follows it while it moves together with the interface.

To summarize, our experiments show how reach and complicated is the behaviour of a particle in a moving $N I$-interface. It is influenced by the shape and speed of the interface and by the director distribution close to the isotropic meniscus. The director orientation, in turn, depends on its anchoring at the interface and cell substrates, as well as on the shape and speed of the interface. Determination of the factors, which control the particle movement, is necessary to develop precise methods of particles and spacers manipulation in LC suspensions. 


\section{REFERENCES}

[1] Pohl, H. A. (1951). J. Appl. Phys. 22, 869.

[2] Pohl, H. A. (1978). Dielectrophoresis. Cambridge University Press: Cambridge.

[3] Markx, G. H. Dyda, P. A., \& Pething, R. (1996). J. Biotechnol., 51, 175.

[4] Washizu, M. \& Kurosava, O. (1990). IEEE Trans. Ind. Appl., 26, 1165.

[5] Morgan, H., Hughes, M. P., \& Green, N. G. (1999). Biophysical J., 77, 516.

[6] Zehner, R., Amundson, K., Knaian, A., Zion, B., \& Johnson, M. (2003). SID 03 Digest, 842.

[7] Ogawa, M., Takahashi, T., Saito, S., Toko, Y., Iwakura, Y., Kobayashi, K., \& Akahane, T. (2003). SID 03 Digest, 584.

[8] Yoshinaga, K., Chiyoda, M., Yoneda, A., Nishida, H., \& Komatsu, M. (1999). Colloid Polym. Sci., 277, 479-482.

[9] Kralchevski, P. et al., (1994). J. Phys. Condens. Matter, 6, A395.

[10] West, J. L., Glushchenko, A., Liao, G., Reznikov, Yu., Andrienko, D., \& Allen, M. P. (2002). Phys. Rev. E, 66, 012702.

[11] Oswald, P., Bechhoefer, J., \& Libchaber. (1987). Phys. Rev. Lett., 58(22), 2318.

[12] Blinov, L. M. \& Chigrinov, V. G. (1994). Electrooptic Effects in Liquid Crystal Materials. Springer-Verlag Inc.: New York.

[13] Palffy-Muhoray, P., Dunmur, D.A., \& Price, A. (1982). Chem. Phys. Lett., $93(6), 572$.

[14] Martire, D. E., Oweimreen, G. A., Argen, G. I., Ryon, S. S., \& Peterson, U. T. (1976). J. Chem. Phys., 66(4), 1456.

[15] Reznikov, Yu., Reshetnyak, V., \& Yaroshchuk., O. (1992). Sov. Phys. JETP, 74 (5), 816.

[16] Wen, B., Kim, J., Yokoyama, H., \& Rosenblatt, C. (2002). Phys. Rev. E, 66, 041502.

[17] Faetti, S. \& Palleschi, V. (1984). Phys. Rev. A, 30, 3241.

[18] Lavrentovich, O. D. (1992). Phys. Rev. A, 46(2), R722.

[19] Voloschenko, D., Pishnyak, O. P., Shiyanovskii, S. V., \& Lavrentovich, O. D. (2002). Phys. Rev. E, 65, 060701(R). 